\title{
Mathematics Is a Sort of Technology
}

\author{
Xiong Zhang \\ Department of Mathematics and Physics, Shaanxi Institute of Education \\ Xi' an 710061, China
}

Tel: 86-29-8153-0123 E-mail: zhangxiong799@yahoo.com.cn

\begin{abstract}
Mathematics has double characters including science and technology. What sort of technology the mathematics is? The direct research object of mathematics is mode, and the mode is the carrier of information, and the evolvement of mode is the process of information processing and information conversion. Therefore, mathematics is the technology of information processing and information conversion.
\end{abstract}

Keywords: Mathematical mode, Information carrier, Mathematical activities, Information processing, Information conversion, Mathematical technology

Mathematics has been regarded as pure and rational science for a long time, but comparing with other natural sciences, the mathematics science has its unique characters. Many subjects such as physics, chemistry, and life science generally are theories or laws of theoretical generalization about certain natural law based on experiments, but mathematics is the mode coming from abstract thinking which can be evolved. With the development of mathematics and the change of social mathematical demands, the characters of mathematics are also changing. In the day of informationization and high technology, people have more realized that mathematics is not only the science, but also the technology. Academician of American Academy of Sciences, J. C. Glimm said that "mathematics is very important for the economic competition, and it is a sort of pivotal and universal technology which can offer ability for people".

Mathematics is regarded as a sort of technology, but what sort of technology it is?

\section{High and new technology is a sort of mathematical technology in nature}

In 1981, American Council of State called experts in mathematics science and relative subjects together and founded a special committee, and though three years' observation and analysis, this committee put forward the report of "further flourishing the mathematics of US". The report pointed out that "the appearance of high technology has pushed our society into the new time of mathematical engineering technology". The president of the special committee, the applied mathematician, E. David said that "only few people can realize that the high technology is a sort of mathematics technology in nature".

Since the second half of the 20th century, the technology character of mathematics is more and more dominant, and the new and high technology more and more depends on the mathematics technology. There are three causes. First, the new and high technology generally has high science and technology content, and generally speaking, the high science and technology degree has high abstraction degree, and the possibility to apply mathematics to solve problems is higher. Second, with the development of modern applied mathematics, mathematics fully presents powerful function and technology power when solving human and nature problems. Third, because of the appearance of computer, the fourth peak of mathematics occurs, i.e. the information-based mathematics. In the time of information, mathematics could be everywhere, and it becomes into the universally applied technology.

For example, the Nobel Prize in medicine of 1979 was awarded to American Cormack and British Hounsfield, and praised that they designed the computed tomography (CT) by the mathematical Radon Transform principle. The basic process of preventing CT can be descried as follows: it can not directly confirm the absorption quantity of x-rays on each point in the 3D space (such as brain scanning), but Cormack regarded this problems as the solution of the tri-function defined on $\mathrm{D}$, and the instrument can measure the average absorption quantity of x-ray on any line segment 1 on $\mathrm{D}$, (it can be denoted by definite integral), and the problem is that when many different values of are measured, whether $\mathrm{F}$ can be confirmed completely. According to the theory of Radon Transform in mathematics, F can be confirmed completely, so CT is invented in principle. The CT which can benefit human beings can be regarded as an implementation of mathematical technology.

For another example, before 1990s, Japan is the big country of TV. To develop the TV mode with high definition, Japan and Western European countries invested billions dollars in the simulated mode. However, in 1993, after the digitalized TV plan of US was pushed, the simulated mode plan immediately became into a piece of waster paper. The mathematical technology supporting TV digitalization is the wavelet technology, and it can compress enormous data to the minimum, and realize the digital transfer of images. In this way, the leading right of world TV industry in the 21 st century is grasped in US. The positioning of plane needs the Kalman filtering technology when the plane takes off and touches down, the auto design needs the computer simulation, the enterprise management needs the mathematical model, the drug effect 
depends on the statistics analysis, the fluctuation of stock needs mathematical estimation, the software design depends on the disperse mathematics, the base of $\mathrm{AI}$ is the mathematical method, the computer simulation technology makes the plane design need not all be implemented in the wind tunnel, the big gun performance test needs not adopt ball firing, and underground nuclear test such as A-bomb can be simulated in computer, and the war game even can be implemented in computer.

Incalculable facts have proved scientists' judgments, "the base of high and new technology is the applied science, and the base of applied science is the mathematics", "high technology is a sort of mathematical technology in nature", "in a sense, the modernization is the mathematization".

\section{Characters of operation, instrument, and invention of mathematics}

By analyzing the dynamic process of mathematics converting from theory and method to technology, it is obvious that the formation between mathematics and technology is not "either-or", but "both-and". Technology is a human rational activity taking efficiency as target, and its target is to design and invent the process, program, instrument and products which don't exist in the nature but are required by people. Science is the knowledge about the object is "what" and "why it is", and technology is the method and measure about "what to do" and "how to do", both are rational activities, but the sense in technology "emphasizes the logic, practical and disciplined method which can solve problems to deal with subjective matters, and it depends on computation, exactness, measurement and system concepts, ". Mathematics is generally thought as the abstract quantitative mode of the nature, and it is not only the explanation of the nature, but the method and measure to engage in other technical activities, which can mainly confirm the rationality of the technology. The confirmation of mathematics in the efficiency and rationality of technology indicates an important advancement of human sense. Just as Weber said, except that the computation could grasp all powers, there was not any one misery power to function. For example, Urbain Le Verrier found the Neptune by computation in 1946. In addition, Newton's law of gravity and Einstein theory were established all depending on mathematics. The utilization of mathematical methods such as computation and modeling could solve kinds of practical problems and technical problems, which fully proves that mathematics is a kind of universal technology of information processing and conversion.

Mathematics is science, but science knowledge will not be pure and absolute for ever, and it contains practical histories and practices, and the technology needs to play the media between the fact and science. Mathematics is an instrumental subject, and it can not only be understood, but also has certain character of operation, especially, it comes down to the establishment skill and operation law of mathematical mode, and the mathematical contents full of inventions such as positive and negative numbers, decimal system, Gauss-Legendre's least square method, Riemann surface of multi-valued complex function uniformization, calculus invented by Newton and Leibniz, and computer, and these contents all have obvious technology component.

The appearance of computer rapidly spreads the modern mathematics to various domains in modern society by the mode of technology, and many people think that the appearance of high technology has pushed our society into the new time of mathematical technology. In modern physics, a series of new phenomena didn't occur in the lab or high-energy accelerator, but were found by the computer. For example, in 1963, the strange attractor was discovered, and the chaos phenomenon of the conservative system was discovered in 1964, and the solution was discovered in 1965, and the bifurcation and the universality of the overfall model were discovered in 1978, which all owned to the scientific computation of computer.

Technology includes the technology with knowledge form and the technology with materialized form, and mathematics is a sort of technology with knowledge form. Without mathematics, high and new technology can not be composed and accumulated, and without high and new technology, mathematics will lose the dwelling place, and can not be developed. For example, without the mathematical theory of time sequence, there is not the oil geography exploitation industry with large scale nowadays. Any one plane or space ship needs to install the automatic navigation equipment designed according to the Kalman Filtering Theory in mathematics. The wing form of Boeing-767 was obtained according to the new approximate solution and computation geometric method of the nonlinear PDE. The main theoretical base of the $\mathrm{x}$-ray technology which pushed the revolution in medicines and medical diagnosis technology is the Radon transform in mathematics. The extension and application of the optimized method and the orthogonal experiment designing method have promoted the enterprise management rule and the technology reform in China.

In addition, the character of technology of mathematics is also represented that it was invented by human being. Mathematics is the abstract description of the nature, and though it accords with the objective rules, but the mathematical contents are not objective and real things in the nature, and they are the abstract mode of the objects invented by mathematicians under the law of scientific thinking. In a word, these three characters of mathematics all accord with the meaning of technology, so when affirming the character of science of mathematics, the character of technology of mathematics can not be denied.

\section{Mathematics is the technology of information processing and information conversion}

Mathematics is the abstract description of objective practice, and its result certainly is the abstract mode. The main 
characters of mode include "formalization" and "generalization". The formalization means that the research object of mathematics is divorced from the actual background to certain meaning, and it is not the concrete review of natural things and it needs not to compare with physical experiences, but it only studies the structure form of a sort of things relationship on general meaning. The generalization indicates the universality and catholicity of the thing reflected by the mode. Both the formalization and the generalization reflect that the mode is the carrier of information. The large numerous of concrete and practical contents in mathematical mode, or certain concrete things are the information contained in the mode. In reverse, the formalized mode is just the carrier of large numerous of information which are processed collectively.

Information is contained in the mode carrier, and any mathematical mode is the carrier of information. If it is not that, mathematics will lose its all complete meanings, but only dead symbols, and in the interior of mathematics, there is not any axiom, and before mathematical mode, there is not any idea.

A series of mathematical activities including the establishment, composing and conversion of mathematical mode are the process of mode deduction. Though mathematical mode is the carrier of information, so the deduction of mode is the process of information processing and information conversion. Therefore, mathematics is the technology of information processing and information conversion.

In this way, whether the mathematical model used by engineers even the mathematical method in science technology, or the deduction adopted by mathematicians to solve mathematical problems even any one small transformation in the mathematical deduction process, all can be summarized as the mathematical technology in nature.

For example, Belgium physicist J. Plateau put forward a problem in 1847, i.e. in a spatial closed curve C, whether the curve face with minimum area and in $\mathrm{C}$ can be found? This problem has been defined as the Plateau Problem. In 1873, in the book of "Experiment and Theory of Hydrostatics in the Single-molecule Model", Plateau pointed out that if people emerged the wire with the closed curve form into the glycerite or suds, and then took the wire out, and there would surely be the membrane with minimum curve face on the wire. In this way, Plateau utilized the principle of liquid surface tension to solve this problem, but it was not satisfactory for mathematicians. In the 19th century, many mathematicians including Riemann and Karl Weierstrass tried to use mathematical method to solve this problem, but none of them succeed. About 60 years later, US mathematician J. Douglas accidentally published the thesis of "the solution of Plateau problem" in Jan of 1931, and he regarded the Plateau problem as the first boundary value problem of a nonlinear ellipse PDE, and solved this problem in the range of generalized solution. Because of this thesis, Douglas obtained the first Fields Medal in 1936. So the key of Douglas' success is an important reduction, i.e. the technology of information conversion.

Each step of the reduction process is to implement the mathematical reduction formally, and its essential is to continually implement information processing or information conversion.

In a ward, the research object of mathematics is abstract mode, and the mode is the carrier of information, and the mathematical activity is the reduction of mode, and the mode reduction is the process of information processing and information conversion. Therefore, mathematics is the technology of information processing and information conversion. Generally speaking, the science technology or the mathematical method universally utilized in other domains is a sort of mathematics technology in fact. The implementation process of mathematics technology mainly includes five main stages (seen in Figure 1).

\section{References}

Zhang, Xiong. (2000). Mathematics: The Technology of Information Processing \& Information Transforming. Studies in Dialectics of Nature, No. 8. 


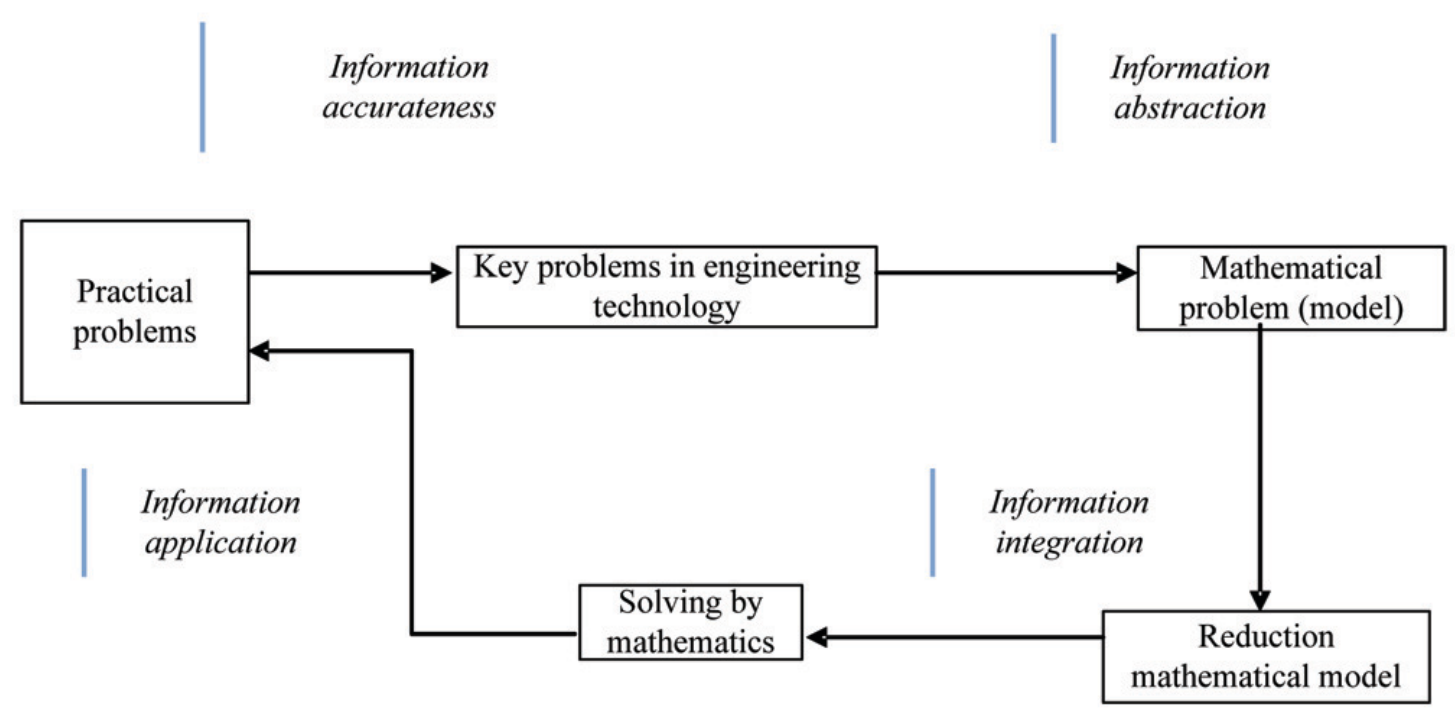

Figure 1. Five Stages of the Implementation Process of Mathematics Technology 